\title{
Benchmarking im Gesundheitswesen: Möglichkeiten und Grenzen
}

\author{
Ausrichtung der Organisationstätigkeiten an anderen, vorzugsweise den besten \\ Organisationen einer Kategorie - das bedeutet «Benchmarking». Vielversprechend \\ erscheinen z. B. Ansätze der Patientenklassifikation, bei der Patienten nach bestimm- \\ ten Merkmalen kategorisiert und zusammengefasst werden.
}

OlafBornemeier

Korrespondenz: Dr. Olaf Bornemeier Hans-Nolte-Strasse 1 D-32429 Minden Tel. 00495717902011

olaf.bornemeier[at] muehlenkreiskliniken.de
Kostendruck und Wettbewerb zwischen den Anbietern von Gesundheitsleistungen werden angesichts der demographischen Entwicklung, medizinischtechnischen Möglichkeiten und begrenzt zur Verfügung stehenden Finanzierungsressourcen zunehmen. In diesem Kontext gewinnen für die Betreiber von Gesundheitseinrichtungen Instrumente des betriebswirtschaftlichen Managements an Bedeutung. Neben Konzepten wie Lean- oder Total Quality Management ist Benchmarking eines dieser Instrumente.

Benchmarking wurde erstmals 1979 durch den Hersteller von Fotokopiergeräten Rank Xerox durchgeführt und hat die externe Orientierung zu einem Prinzip erhoben. Es wird charakterisiert als «Ausrichtung der Organisationstätigkeiten an anderen, vorzugsweise den besten Organisationen einer Kategorie». Dieser Ansatz setzt die tendenzielle Gleichartigkeit der Anforderungen an eine Organisation und die Existenz allgemeingültiger Erfolgsfaktoren voraus. Er beinhaltet somit notwendigerweise die Abstraktion vom Besonderen der Organisationsmerkmale, sonst wäre die Übernahme von Praktiken, die für andere Organisationen Wettbewerbsvorteile erwirkt haben, wertlos.

Benchmarking ist die Auswahl und Realisierung einer spezifischen Handlungsalternative zur Lösung eines bestehenden Problems. Anstatt für erkannte Probleme eine eigenständige Lösung zu entwickeln, werden als besser bewertete Lösungen anderer nachgeahmt und adaptiert. Vergleiche stellen hierbei gemessene Leistungsausprägungen sowie konkrete Verfahrensbeschreibungen einander gegenüber, um Unterschiede und Identitäten zu identifizieren. Die Imitation jedoch ist das eigentlich Neue am Benchmarking-Ansatz. Sie kennzeichnet die Nachahmung und ggf. Adaption von an einem anderen Ort gefundenen Praktiken zur Lösung von Aufgabenstellungen.

Zur Lösung von Problemen stehen grundsätzlich drei Reaktionsmuster zur Auswahl. Erstens können bisherige Verhaltensweisen beibehalten und das Problem kann ignoriert werden. Zweitens können eigenständig neue Handlungsmuster entwickelt und implementiert werden. Dieser «innovative» Ansatz ist je- doch mit Risiken verbunden. Drittens schliesslich können bereits bewährte Verhaltensmuster nachgeahmt werden. Dies geht regelhaft einher mit einer Verringerung von Risiken oder aber ergibt gegenüber dem eigenständigen Ansatz Zeit- und Kostenvorteile. Weil es infolge beschränkter Potentiale nahezu unmöglich ist, auf allen organisatorischen Gebieten innovatorisch tätig zu sein, stellt die Imitation eine ernsthafte Handlungsalternative dar.

Aus der Perspektive desjenigen, der imitiert werden soll, stellt sich die Situation jedoch anders dar. Im Falle eines indifferenten Verhältnisses kann eine Nachahmung sofort Zustimmung finden. Existieren jedoch interdependente oder sogar rivalisierende Beziehungen, kann der Wunsch nach Imitation nur positiv beschieden werden, wenn sich auch für den Nachgeahmten Vorteile ergeben. Dies ist dann der Fall, wenn etwa durch die Zusammenarbeit weitere Konkurrenten ausgeschaltet oder Wettbewerbsabstände vergrössert werden können.

Seit 1990 werden auch im Gesundheitswesen Benchmarking-Projekte mit beachtlichem Erfolg durchgeführt. Hierbei ist zu beachten, dass das Leistungsgeschehen innerhalb der Gesundheitsversorgung komplex und sehr differenziert ist. Dies findet seinen Ausdruck in der Vielzahl unterschiedlicher Gesundheitseinrichtungen und in der Bekämpfung von ca. 60000 Krankheitsbildern. Zusätzlich weist jeder Patient individuell spezifische Eigenschaften auf, so dass die Dienstleistungen den Anforderungen des Einzelnen angepasst werden müssen. Die Schlussfolgerung, dass die Bewertung und Beurteilung von Einrichtungen der Gesundheitsversorgung nicht möglich sei, wäre jedoch falsch. Allerdings sind einige Merkmale zu beachten.

Sofort einsichtig ist, dass Vergleiche von grossen Einheiten wie etwa Krankenhäuser oder Arztpraxen in ihrer Gesamtheit wenig aussagekräftig in Bezug auf Praktiken sein können. Im Fokus solcher Überlegungen standen daher bisher Versuche, diese Einheiten etwa mittels morphologischer oder mathematischer Verfahren «vergleichbar» zu machen. Sowohl die Grösse als auch die Heterogenität verhindern jedoch das Auffinden bester Praktiken. 


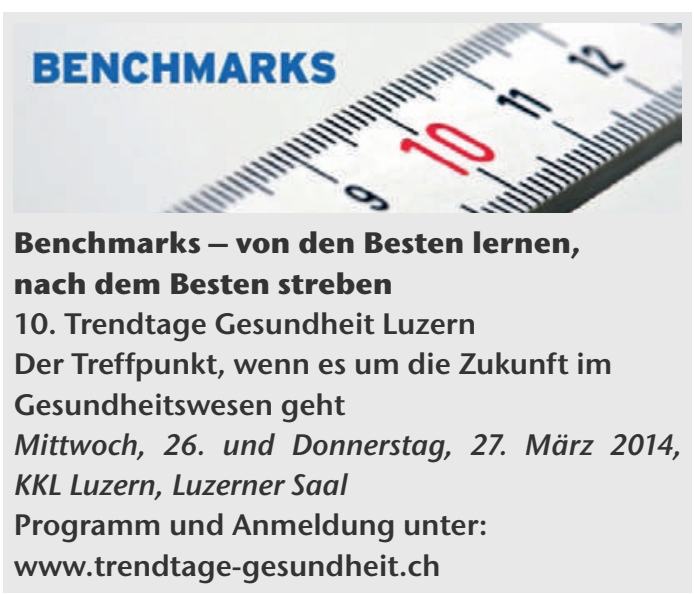

Vielversprechender erscheinen Ansätze der Patientenklassifikation, mit deren Hilfe Patienten nach bestimmten Merkmalen kategorisiert und zusammengefasst werden. Patientenklassifikationssysteme lassen sich nach dem Fokus der Betrachtung unterscheiden. Die Mehrheit der Systeme betrachtet ausschliesslich medizinische Belange, die Merkmale wie etwa Überlebensprognose, medizinische Behandlungsschwierigkeiten, Dringlichkeit einer therapeutischen Intervention oder Behandlungsstadien beinhalten.

Andere Systeme kombinieren medizinische Faktoren mit Faktoren, die den Ressourceneinsatz betreffen. Die bekanntesten sind natürlich die Diagnosis Related Groups (DRGs) für den stationären Sektor oder aber Ambulant Patient Groups (APGs) für den ambulanten Bereich. Auch sind Ansätze zur sektorenübergreifenden Episodengruppierung wie etwa Adjusted Clinical Groups oder Clinical Risk Groups vorhanden. Die genannten Ansätze haben erkannt, wie wichtig die initiale Schwere der Erkrankung sowie die Behandlungsmöglichkeiten sowohl in medizinischer Hinsicht als auch im Hinblick auf die Inanspruchnahme von Ressourcen ist. Allerdings arbeiten die Klassifizierungssysteme unter der Prämisse der Gleichartigkeit des Outputs. Aufgrund der Koppelung medizinischer und ökonomischer Aspekte bei der Gruppenbildung besteht immer noch eine gewisse Grobheit, die verhindert, dass keine signifikanten Informationen darüber gewonnen werden können, wo innerhalb des Patientenbehandlungsprozesses eine Verbesserung anzusetzen ist. Das Vorhandensein einer Schwäche lässt sich jedoch wirkungsvoll auf dieser Ebene beschreiben. In einem Vergleich der Inputgrössen (z. B. Kosten oder Zeitparameter) einer Gruppe mit der von Wettbewerbern tritt die eigene relative Position offen zutage. Vergleiche auf dieser Ebene sind daher gutgeeignet, um Prioritäten zu setzen und um Häuser mit der besten Praktik in dieser Gruppe anhand des Ergebnisses zu identifizieren.

Um allerdings den Problemlösungsanspruch des Benchmarkings einlösen zu können, bedarf es weiterer Verfeinerungen. Erstens verlangt die Imitation von besten Praktiken den Erwerb von Know-how über einzelne Tätigkeiten. Zweitens bietet eine den Strukturen und Produkten verhaftete Denkweise kaum Möglichkeiten, die eigene Branche zu verlassen. Somit ist die Beschreibung der Leistungserstellung aus einer prozessorientierten Perspektive relevant. Um die auf verschiedene Aufgabenträger verteilte Arbeit zu vergleichen und bessere Praktiken zu übernehmen, ist die Prozesssicht eine praktikable Basis.
Der Behandlungsprozess ist vom Krankheitsverlauf des Patienten geprägt. Daher ist eine medizinische Sicht auf diesen Prozess unumgänglich. Darüber hinaus sind jedoch ablauforganisatorische Betrachtungsweisen erforderlich. Sowohl medizinische Merkmale der Therapie, Diagnostik und Pflege als auch ablauforganisatorische Gestaltungsmassnahmen bestimmen das Leistungsniveau und die Effizienz des zu analysierenden Geschehens. Ärztliches Handeln kann sicherlich kein ausschliesslich datengesteuerter Akt sein. Gleichwohl besteht die Notwendigkeit, die Varianz ärztlichen Handelns auf innermedizinisch geleitete Kriterien zurückzuführen. Eine Hilfestellung hierfür sind auf der Basis der Evidence based medicine entwickelte Leitlinien. Patientenpfade hingegen sind der Versuch, die am Patienten durchzuführenden Massnahmen in ihrem Ablauf zu erfassen. Die Leitlinie gibt an, was gemacht wird. Der Patientenpfad zeigt dagegen, wann und wo etwas gemacht wird. Die vergleichende Analyse von beschriebenen Behandlungen, ausgehend von Symptomen oder Diagnosen ermöglicht die Aufdeckung qualitätsrelevanter und wirtschaftlicher Optimierungspotentiale. In den Vereinigten Staaten von Amerika wurde auf dieser Basis ein Benchmarking durchgeführt. Disease Management Programme für Asthmaerkrankungen, die den sektorenübergreifenden Patientenpfad darstellen, wurden einander gegenübergestellt. Es konnte gezeigt werden, dass Notfallbehandlungen und Hospitalisierungen minimal sind, wenn die Routinekontrollen von Fachärzten statt von Allgemeinärzten durchgeführt werden.

Von der Theorie der Ablauforganisation ausgehend lassen sich die auch in der Gesundheitsversorgung auftretenden grundlegenden Problemtypen Arbeitsverteilung, Gruppierung, Reihenfolge, Leistungsabstimmung, Transport und Standort antreffen. Im Kontext des Benchmarking lassen sich diese Problemtypen als generellster Ansatz zur Ausdifferenzierung von Aufgabenstellungen verstehen. Zwingend vorgeschriebene Verfahren, von denen nicht abgewichen werden kann, können nun als Nebenbedingungen bzw. Restriktion der allgemeinen Problemtypen interpretiert werden. Hiervon ausgehend lassen sich auch Teilprozesse wie etwa die administrative Aufnahme, die medizinische Aufnahme, die Operation, Visiten usw. analysieren. Auf diese Weise lassen sich auch für die Erbringer von Gesundheitsleistungen Benchmarking-Partner ausserhalb der Branche finden. Die Mühlenkreiskliniken haben z.B. zur Reorganisation des Hol- und Bringdiensts Praktiken von Taxiunternehmen übernommen.

Benchmarking kann ein relevanten Beitrag zur Verbesserung organisatorischer Praktiken sein. Der Nutzen kann dabei umso grösser eingeschätzt werden, je eher es gelingt, die Branche zu verlassen und die Übernahme «fremder» Problemlösungen zu akzeptieren. 